\title{
ENERGY-MOMENTUM TIME INTEGRATION OF GRADIENT-BASED MODELS FOR FIBER-BENDING STIFFNESS IN ANISOTROPIC THERMO-MECHANICAL CONTINUA
}

\author{
JULIAN DIETZSCH ${ }^{*}$, MICHAEL GROSS ${ }^{\dagger}$ AND INIYAN KALAIMANI ${ }^{\dagger \dagger}$ \\ Technische Universität Chemnitz \\ Professorship of applied mechanics and dynamics \\ Reichenhainer Straße 70, D-09126 Chemnitz \\ webpage: https://www.tu-chemnitz.de/mb/TMD \\ email: * julian.dietzsch@mb.tu-chemnitz.de, \\ † michael.gross@mb.tu-chemnitz.de, \\ †† iniyan.kalaimani@mb.tu-chemnitz.de
}

Key words: Fiber-bending stiffness, fiber-reinforced materials, locking behavior, mixed finite elements, mixed variational principle, gradient thermoelasticity.

\begin{abstract}
Accurate dynamic simulations of 3D fiber-reinforced materials in lightweight structures motivate our research activities. In order to accomplish this, the material reinforcement is performed by fiber rovings with a separate bending stiffness, which can be modelled by a second-order gradient of the deformation mapping (see Reference [10]). With an independent field for the gradient of the right Cauchy-Green tensor, we extend the thermoelastic Cauchy continuum for fiber-matrix composites with single fibers. In addition, we use accurate higherorder energy-momentum schemes in combination with mixed finite element methods to obtain numerically stable long-term dynamic simulations and locking free meshes. Therefore, we introduce additional independent fields of well-known as well as new mixed finite elements within a variational-based space-time finite element method and adapt it to the new material formulation. We use Cook's cantilever beam as representative numerical example. We primarily analyze the influence of the fiber bending stiffness as well as the spatial and time convergence up to cubic order, but also look at the influence of Fourier's heat conduction in the matrix and fiber families.
\end{abstract}

\section{CONTINUUM MODEL}

As continuum model, we consider an anisotropic material with the fiber roving direction $\boldsymbol{a}_{0}$, moving in the Euclidean space $\mathbb{R}^{n_{\mathrm{dim}}}$ with the constant ambient temperature $\Theta_{\infty}$. The strain 
energy function of the material with a thermoelastic matrix and fiber roving is given by

$$
\Psi\left(\boldsymbol{C}, \Theta, a_{0}\right)=\Psi_{\mathrm{M}}(\boldsymbol{C}, \Theta)+\Psi_{\mathrm{F}}\left(\boldsymbol{C}, \Theta, a_{0}\right)+\Psi_{\mathrm{HOG}}^{\mathrm{X}}\left(\ldots, a_{0}\right),
$$

which is split into a matrix part $\Psi_{\mathrm{M}}$ an fiber roving part $\Psi_{\mathrm{F}}$ and an higher order gradient part $\Psi_{\mathrm{HOG}}^{\mathrm{X}}$. Here $\boldsymbol{F}$ define the deformation gradient, $\boldsymbol{C}=\boldsymbol{F}^{T} \boldsymbol{F}$ define the right Cauchy-Green tensor and $\Theta$ define the absolute temperature. With the volume dilatation $J(\boldsymbol{C})=\operatorname{det}[\boldsymbol{F}]=\sqrt{\operatorname{det}[\boldsymbol{C}]}$, we assume the specific dependencies

$$
\begin{aligned}
\Psi_{\mathrm{M}}(\boldsymbol{C}, J, \Theta) & =\Psi_{\mathrm{M}}^{\mathrm{iso}}(\boldsymbol{C}, J)+\Psi_{\mathrm{M}}^{\mathrm{vol}}(J)+\Psi_{\mathrm{M}}^{\mathrm{cap}}(\Theta)+\Psi_{\mathrm{M}}^{\text {coup }}(\Theta, J) \\
\Psi_{\mathrm{F}}\left(\boldsymbol{C}, \Theta, a_{0}, \ldots\right) & =\Psi_{\mathrm{F}}^{\mathrm{ela}}\left(\boldsymbol{C}, \boldsymbol{a}_{0}\right)+\Psi_{\mathrm{F}}^{\mathrm{cap}}(\Theta)+\Psi_{\mathrm{F}}^{\mathrm{coup}}(\Theta, \boldsymbol{C})
\end{aligned}
$$

The elastic part of the matrix function $\Psi_{M}$ is split into an isochoric part $\Psi_{M}^{\text {iso }}$ and a volumetric part $\Psi_{\mathrm{M}}^{\mathrm{vol}}$. We subdivided the thermo-elastic free energy of the matrix material into a heat capacity part $\Psi_{\mathrm{M}}^{\mathrm{cap}}$ and the part of the thermo-mechanical coupling effect $\Psi_{\mathrm{M}}^{\text {coup }}$, where $\beta_{\mathrm{M}}$ ist the coefficient of linear thermal expansion for the matrix. The thermal part of the fiber roving free energy is separated in the same manner. We consider a heat capacity function $\Psi_{\mathrm{F}}^{\mathrm{cap}}$ and the function of the thermo-mechanical coupling $\Psi_{\mathrm{F}}^{\mathrm{coup}}$ with the coefficients of linear thermal expansion $\beta_{\mathrm{F}}$, the structural tensor $\boldsymbol{M}=\boldsymbol{a}_{0} \otimes \boldsymbol{a}_{0}$ and the fourth invariant $I_{4}=\operatorname{tr}[\boldsymbol{C M}]$. Both coupling parts are given by

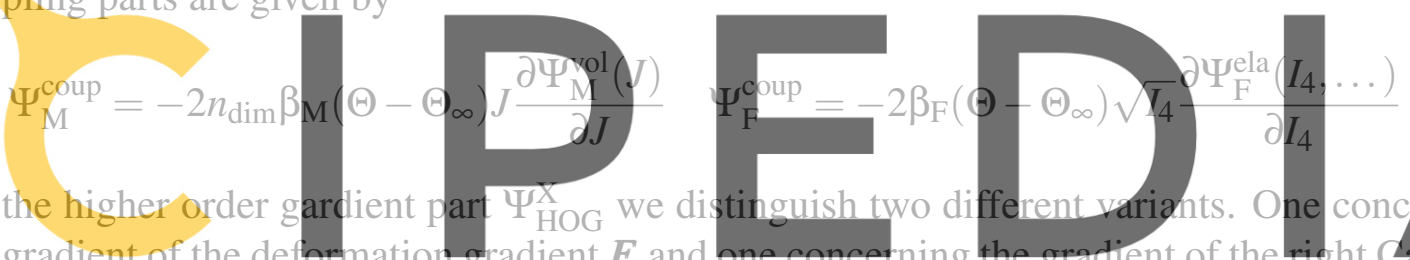

the gradient of the deformation gradient $F$ and

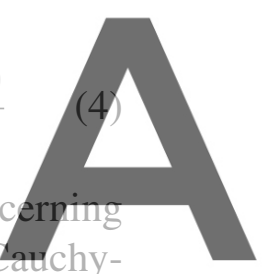

Green tensor $C$. This part capture the bending of the fiber roving, during $\Psi_{\mathrm{F}}^{\mathrm{ela}}$ considers the

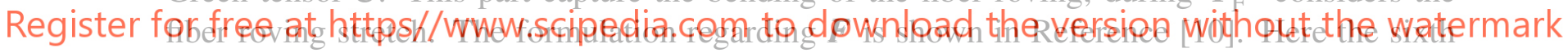
and seventh invariants are given by

$$
I_{6}^{\mathrm{F}}(\boldsymbol{F}, \nabla \boldsymbol{F})=\boldsymbol{\kappa}_{0}^{\mathrm{F}} \cdot \boldsymbol{\kappa}_{0}^{\mathrm{F}} \quad I_{7}^{\mathrm{F}}(\boldsymbol{F}, \nabla \boldsymbol{F}, \boldsymbol{C})=\boldsymbol{\kappa}_{0}^{\mathrm{F}} \cdot \boldsymbol{C} \cdot \boldsymbol{\kappa}_{0}^{\mathrm{F}} \quad \boldsymbol{\kappa}_{0}^{\mathrm{F}}=\boldsymbol{\Lambda}^{\mathrm{F}} \cdot \boldsymbol{a}_{0}
$$

with the referential representation

$$
\Lambda^{\mathrm{F}}(\boldsymbol{F}, \nabla \boldsymbol{F})=\boldsymbol{F}^{T} \cdot \boldsymbol{a}_{0} \cdot \nabla \boldsymbol{F}^{T}
$$

For the seventh invariant, it is important to note here that $I_{7}$ is depend on $\boldsymbol{C}$ as well as $\boldsymbol{\Lambda}$. Thus, for the strain energy function of the higher order gradient, the dependencies are

$$
\begin{aligned}
\Psi_{\mathrm{HOG}}^{\mathrm{F}}\left(\boldsymbol{F}, \nabla \boldsymbol{F}, \boldsymbol{C}, \boldsymbol{a}_{0}\right) & =f\left(I_{6}^{\mathrm{F}}(\boldsymbol{F}, \nabla \boldsymbol{F}), I_{7}^{\mathrm{F}}(\boldsymbol{F}, \nabla \boldsymbol{F}, \boldsymbol{C})\right) \\
\Psi_{\mathrm{HOG}}^{\mathrm{F}}\left(\boldsymbol{\Lambda}^{\mathrm{F}}, \boldsymbol{C}, \boldsymbol{a}_{0}\right) & =\hat{f}\left(I_{6}^{\mathrm{F}}\left(\boldsymbol{\Lambda}^{\mathrm{F}}\right), I_{7}^{\mathrm{F}}\left(\boldsymbol{\Lambda}^{\mathrm{F}}, \boldsymbol{C}\right)\right)
\end{aligned}
$$

In Reference [11] a variant of the higher order gradient formulation in $\boldsymbol{C}$ is shown. From this we derive the following formula for the sixth invariant

$$
I_{6}^{C}(\nabla \boldsymbol{C})=\left(\boldsymbol{a}_{0} \cdot \nabla \boldsymbol{C} \cdot \boldsymbol{a}_{0}\right) \cdot\left(\boldsymbol{a}_{0} \cdot \nabla \boldsymbol{C} \cdot \boldsymbol{a}_{0}\right)
$$


If we now set

$$
\Lambda^{\mathrm{C}}(\nabla \boldsymbol{C})=\boldsymbol{a}_{0} \cdot \nabla \boldsymbol{C}
$$

we get the same expressions for the invariants as for $\boldsymbol{F}$, given by

$$
I_{6}^{\mathrm{C}}(\nabla \boldsymbol{C})=\mathbf{k}_{0}^{\mathrm{C}} \cdot \mathbf{\kappa}_{0}^{\mathrm{C}} \quad I_{7}^{\mathrm{C}}(\boldsymbol{C}, \nabla \boldsymbol{C})=\mathbf{k}_{0}^{\mathrm{C}} \cdot \boldsymbol{C} \cdot \mathbf{k}_{0}^{\mathrm{C}} \quad \mathbf{k}_{0}^{\mathrm{C}}=\boldsymbol{\Lambda}^{\mathrm{C}} \cdot \boldsymbol{a}_{0}
$$

and the final dependencies read

$$
\begin{aligned}
& \Psi_{\mathrm{HOG}}^{\mathrm{C}}\left(\nabla \boldsymbol{C}, \boldsymbol{C}, \boldsymbol{a}_{0}\right)=f\left(I_{6}^{\mathrm{C}}(\nabla \boldsymbol{C}), I_{7}^{\mathrm{C}}(\nabla \boldsymbol{C}, \boldsymbol{C})\right) \\
& \Psi_{\mathrm{HOG}}^{\mathrm{C}}\left(\boldsymbol{\Lambda}^{\mathrm{C}}, \boldsymbol{C}, \boldsymbol{a}_{0}\right)=\hat{f}\left(I_{6}^{\mathrm{C}}\left(\boldsymbol{\Lambda}^{\mathrm{C}}\right), I_{7}^{\mathrm{C}}\left(\boldsymbol{\Lambda}^{\mathrm{C}}, \boldsymbol{C}\right)\right)
\end{aligned}
$$

\section{FINITE ELEMENT FORMULATION}

The finite element discretization follows from the mixed principle of virtual power (see Reference $[4,5])$. Here, we need the complete internal energy, which consists of the assumed temperature field $\tilde{\Theta}$, the entropy density field $\eta$ as the corresponding Lagrange multiplier, the superimposed stress tensor $\tilde{S}$ to derive an energy-momentum scheme, an independent mixed field $\tilde{C}$ and the corresponding Lagrangian multiplier $S$. The internal energy functional reads
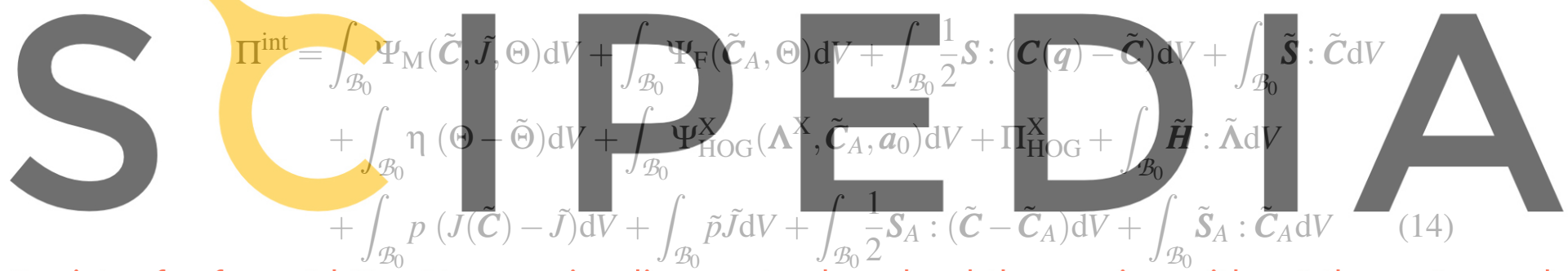

Register for free at https//www.scipedia.com to download the version without the watermark

To avoid locking effects we introduce an independent volume dilatation $\widetilde{J}$ (see Reference [1])

and the field $\tilde{C}_{A}$ (see Reference [2]) for the anisotropic part $\Psi_{\mathrm{F}}$. Here, the Lagrange multiplier

$p$ plays the role of the hydrostatic pressure and the Lagrange multiplier $\boldsymbol{S}_{A}$ represents the stress tensor of the anisotropic part. To obtain an energy-momentum scheme, we also introduce the superimposed pressure $\tilde{p}$ and superimposed stress tensor $\tilde{\boldsymbol{S}}_{A}$. For the higher order gradient fomulation with respect to $\boldsymbol{F}$, we introduce an independent field for $\boldsymbol{F}$, for $\nabla \boldsymbol{F}$ and for $\boldsymbol{\Lambda}_{\mathrm{F}}$

$$
\Pi_{\mathrm{HOG}}^{\mathrm{F}}=\int_{\mathcal{B}_{0}} \tilde{\boldsymbol{P}}:(\boldsymbol{F}-\tilde{\boldsymbol{F}}) \mathrm{d} V+\int_{\mathcal{B}_{0}} \boldsymbol{B} \odot_{3}(\nabla(\tilde{\boldsymbol{F}})-\tilde{\boldsymbol{\Gamma}}) \mathrm{d} V+\int_{\mathcal{B}_{0}} \boldsymbol{H}:\left(\boldsymbol{\Lambda}_{\mathrm{F}}(\tilde{\boldsymbol{F}}, \tilde{\boldsymbol{\Gamma}})-\tilde{\boldsymbol{\Lambda}}\right) \mathrm{d} V
$$

By the independent definition of $\tilde{\boldsymbol{F}}$ and $\tilde{\boldsymbol{\Gamma}}$ it is later in the discrete setting not necessary to construct a double gradient of the spatial shape functions. The introduction of $\tilde{\Lambda}$ is necessary to have an objective quantity for the construction of an energy-momentum scheme. For the higher order gradient fomulation with respect to $\boldsymbol{C}$, we build the functional in the same manner

$$
\Pi_{\mathrm{HOG}}^{\mathrm{C}}=\int_{\mathcal{B}_{0}} \frac{1}{2} \boldsymbol{S}_{G}:\left(\tilde{\boldsymbol{C}}-\tilde{\boldsymbol{C}}_{G}\right)+\int_{\mathcal{B}_{0}} \boldsymbol{B} \odot_{3}\left(\nabla\left(\tilde{\boldsymbol{C}}_{G}\right)-\tilde{\boldsymbol{\Gamma}}\right) \mathrm{d} V+\int_{\mathcal{B}_{0}} \boldsymbol{H}:(\boldsymbol{\Lambda}(\tilde{\boldsymbol{\Gamma}})-\tilde{\boldsymbol{\Lambda}}) \mathrm{d} V
$$


Here, we introduce an independent field for $\boldsymbol{C}$, for $\nabla \boldsymbol{C}$ and for $\boldsymbol{\Lambda}_{\mathrm{C}}$. The further field with respect to $\boldsymbol{C}$ is introduced because $\boldsymbol{S}_{G}$ is assumed to be asymmetric, and therefore no symmetries in the voigt notation are used later in the programming. Furthermore, it can be seen that $\boldsymbol{\Lambda}$ depends only on $\tilde{\Gamma}$, which later leads to a less complex weak form. For both formulations we introduce an superimposed field $\tilde{\boldsymbol{H}}$ to obtain an energy-momentum scheme, as well. The superimposed fields (see Reference [5] and [4]) are given by

$$
\begin{aligned}
& \tilde{\boldsymbol{S}}=\frac{\tilde{\Psi}(1)-\tilde{\Psi}(0)-\int \frac{\partial \Psi_{M}^{\text {iso }}}{\partial \tilde{\boldsymbol{C}}}: \dot{\tilde{\boldsymbol{C}}}-\int \frac{\partial\left(\Psi_{\mathrm{M}}^{\mathrm{cap}}+\Psi_{\mathrm{F}}^{\mathrm{cap}}\right)}{\partial \Theta} \dot{\Theta}}{\dot{\tilde{\boldsymbol{C}}}: \dot{\tilde{\boldsymbol{C}}}} \dot{\tilde{\boldsymbol{C}}} \\
& \tilde{p}=\frac{\tilde{\Psi}(1)-\tilde{\Psi}(0)-\int \frac{\partial\left(\Psi_{\mathrm{M}}^{\text {iso }}+\Psi_{\mathrm{M}}^{\mathrm{vol}}\right)}{\partial \tilde{J}} \dot{\tilde{J}}-\int \frac{\partial \Psi_{\mathrm{M}}^{\text {coup }}}{\partial \Theta} \dot{\Theta}}{\dot{\tilde{J}} \dot{\tilde{J}}} \dot{\tilde{J}} \\
& \begin{array}{l}
\tilde{S}_{A}=\frac{\tilde{\Psi}(1)-\tilde{\Psi}(0)-\int \frac{\partial\left(\Psi_{\mathrm{F}}^{\text {ela }}+\Psi_{\mathrm{HOG}}^{\mathrm{X}}\right)}{\partial \tilde{C}_{A}}: \dot{\tilde{C}}_{A}-\int \frac{\partial \Psi_{\mathrm{F}}^{\text {coup }}}{\partial \Theta} \dot{\Theta}}{\dot{\tilde{C}}_{A}: \dot{\tilde{C}}_{A}} \dot{\tilde{C}}_{A} \\
\tilde{H}=\frac{\tilde{\Psi}(1)-\tilde{\Psi}(0)-\int \frac{\partial \Psi \Psi_{\mathrm{HOG}}^{X}}{\partial \tilde{\Lambda}}: \dot{\tilde{\Lambda}}_{\dot{\Lambda}}}{\tilde{\tilde{\Lambda}}: \dot{\tilde{\Lambda}}}
\end{array}
\end{aligned}
$$

\section{For the mixed princip}

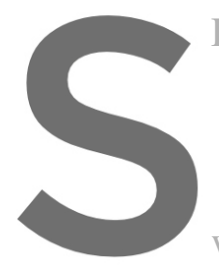

with the velocity $v$, the linear momentum $p$
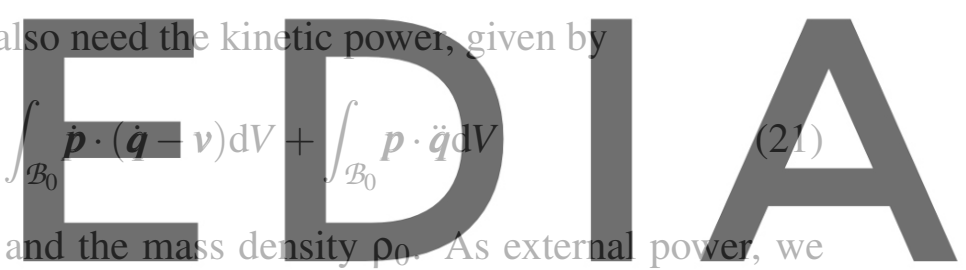

assume

Register for free at https//www.scipedia.com to download the versige

$$
\begin{aligned}
& \dot{\Pi}^{\mathrm{ext}}=-\int_{\partial \mathcal{B}_{0}} \lambda_{q} \cdot\left(\dot{q}-\dot{q}^{\mathrm{ref}}\right) \mathrm{d} A-\int_{\mathcal{B}_{0}} \rho_{0} g \cdot \dot{q} \mathrm{~d} V+\int_{\mathcal{B}_{0}} \nabla\left(\frac{\Theta}{\Theta}\right) \cdot Q \mathrm{~d} V \\
& \boldsymbol{Q}=-\left[J\left(\tilde{\boldsymbol{C}}_{A}\right) \frac{k_{\mathrm{F}}-k_{\mathrm{M}}}{\tilde{\boldsymbol{C}}_{A}: \boldsymbol{M}}+k J(\tilde{\boldsymbol{C}}) \tilde{\boldsymbol{C}}^{-1}\right] \nabla \Theta
\end{aligned}
$$

In this case, $\boldsymbol{Q}$ denotes the Piola heat flux vector derived from Duhamel's law (see Reference [5]), where $k_{\mathrm{M}}$ and $k_{\mathrm{F}}$ denotes the material conductivity coefficients for matrix and fiber roving. Here, $\dot{\boldsymbol{q}}^{\text {ref }}$ denotes the time evolution of a prescribed boundary displacement with the Lagrange multiplier $\boldsymbol{\lambda}_{q}$. The vector $\boldsymbol{g}$ denotes the gravitational force. The total energy balance $\dot{\mathcal{H}}$ thus reads

$$
\begin{aligned}
\dot{\mathcal{H}}=\dot{T} & (\dot{\boldsymbol{q}}, \dot{\boldsymbol{v}}, \dot{\boldsymbol{p}})+\dot{\Pi}^{\mathrm{ext}}\left(\dot{\boldsymbol{q}}, \boldsymbol{\lambda}_{q}, \tilde{\Theta}, \dot{\Theta}\right) \\
& +\dot{\Pi}^{\mathrm{int}}\left(\dot{\boldsymbol{q}}, \tilde{\Theta}, \dot{\boldsymbol{\eta}}, \dot{\tilde{\boldsymbol{C}}}, \dot{\tilde{\tilde{J}}}, \dot{\tilde{\boldsymbol{C}}}_{A}, \boldsymbol{S}, p, \boldsymbol{S}_{A}, \dot{\tilde{\Gamma}}, \dot{\tilde{\boldsymbol{\Lambda}}}, \boldsymbol{B}, \boldsymbol{H}, \ldots\right)
\end{aligned}
$$

The superimposed fields $\left(\tilde{\boldsymbol{S}}, \tilde{p}, \tilde{\boldsymbol{S}}_{A}, \tilde{\boldsymbol{H}}\right)$ as well as the Piola heat flux vector $\boldsymbol{Q}$ are defined as parameter fields and not as arguments. By variation with respect to the variables in the argument of 
Eqn. (24), that is $\int_{T} \delta_{*} \dot{\mathcal{H}} \mathrm{d} t \equiv \int_{T}\left[\delta_{*} \dot{T}+\delta_{*} \dot{\Pi}^{\mathrm{ext}}+\delta_{*} \dot{\Pi}^{\text {int }}\right] \mathrm{d} t=0$, we obtain the total weak forms. First, the weak forms which occur in both variants of the higher order gradient formulation read

$$
\begin{aligned}
& \int_{T} \int_{\mathcal{B}_{0}}\left[\frac{1}{\rho_{0}} \boldsymbol{p}-\dot{\boldsymbol{q}}\right] \cdot \delta \dot{\boldsymbol{v}} \mathrm{d} V \mathrm{~d} t=0 \quad \int_{T} \int_{\partial \mathcal{B}_{0}}\left[-\boldsymbol{\lambda}_{q}\right] \cdot \delta \dot{\boldsymbol{q}} \mathrm{d} A \mathrm{~d} t=0 \int_{T} \int_{\partial \mathcal{B}_{0}}\left[\dot{\tilde{\boldsymbol{q}}}-\dot{\boldsymbol{q}}^{\mathrm{ref}}(t)\right] \cdot \delta \boldsymbol{\lambda}_{q} \mathrm{~d} A \mathrm{~d} t=0 \\
& \int_{T} \int_{\mathcal{B}_{0}}\left[\eta+\frac{\partial \Psi}{\partial \Theta}\right] \delta \dot{\Theta} \mathrm{d} V \mathrm{~d} t=0 \quad \int_{T} \int_{\mathcal{B}_{0}}\left[\frac{\operatorname{Div}[\boldsymbol{Q}]}{\Theta}+\dot{\eta}\right] \delta \tilde{\Theta} \mathrm{d} V \mathrm{~d} t=0 \\
& \int_{T} \int_{\mathcal{B}_{0}} \frac{1}{2}[\dot{\tilde{\boldsymbol{C}}}-\dot{\boldsymbol{C}}]: \delta S \mathrm{~d} V \mathrm{~d} t=0 \quad \int_{T} \int_{\mathcal{B}_{0}}[\Theta-\tilde{\Theta}] \delta \dot{\eta} \mathrm{d} V \mathrm{~d} t=0 \\
& \int_{T} \int_{\mathcal{B}_{0}}[\dot{\tilde{J}}-\dot{J}] \delta p \mathrm{~d} V \mathrm{~d} t=0 \quad \int_{T} \int_{\mathcal{B}_{0}}\left[p-\left[\frac{\partial \Psi}{\partial \tilde{J}}+\tilde{p}\right]\right] \delta \dot{\tilde{J}} \mathrm{~d} V \mathrm{~d} t=0 \\
& \int_{T} \int_{\mathcal{B}_{0}} \frac{1}{2}\left[\dot{\tilde{C}}_{A}-\dot{\tilde{C}}\right]: \delta S_{A} \mathrm{~d} V \mathrm{~d} t=0 \quad \int_{T} \int_{\mathcal{B}_{0}}\left[\frac{1}{2} S_{A}-\left[\frac{\partial \Psi}{\partial \tilde{C}_{A}}+\tilde{S}_{A}\right]\right]: \delta \dot{\tilde{C}}_{A} \mathrm{~d} V \mathrm{~d} t=0
\end{aligned}
$$$$
\int_{T} \int_{\mathcal{B}_{0}}\left[\frac{1}{2} \boldsymbol{S}-\left(\frac{\partial \Psi}{\partial \tilde{\boldsymbol{C}}}+\frac{p}{2 J(\tilde{\boldsymbol{C}})} \operatorname{cof}[\tilde{\boldsymbol{C}}]+\frac{1}{2} \boldsymbol{S}_{A}+\tilde{\boldsymbol{S}}\right)\right]: \delta \dot{\tilde{\boldsymbol{C}}} \mathrm{d} V \mathrm{~d} t=0
$$

$\int_{T} \int_{\mathcal{B}_{0}}\left[\Lambda^{\mathrm{X}}(\ldots)-\tilde{\Lambda}\right]: \delta_{*} H \mathrm{~d} V \mathrm{~d} t=0 \quad \int_{T} \int_{\mathcal{B}_{0}}\left[H-\left[\frac{\partial \Psi}{\partial \tilde{\Lambda}}+\tilde{H}\right]\right]: \delta_{*} \dot{\tilde{\Lambda}} \mathrm{d} V \mathrm{~d} t=0$
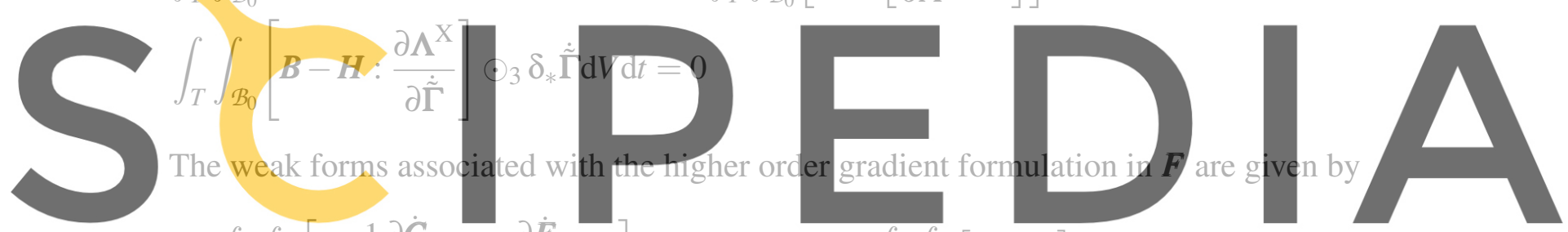

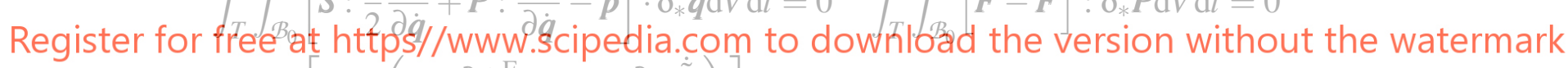

$$
\int_{T} \int_{\mathcal{B}_{0}}\left[\mathbb{P}-\left(\boldsymbol{H}: \frac{\partial \Lambda^{\mathrm{F}}}{\partial \dot{\tilde{\boldsymbol{F}}}}+B \odot_{3} \frac{\partial \nabla \dot{\tilde{F}}}{\partial \dot{\tilde{\boldsymbol{F}}}}\right)\right]: \delta_{*} \dot{\tilde{F}} \mathrm{~d} V \mathrm{~d} t \quad \int_{T} \int_{\mathcal{B}_{0}}[\nabla(\dot{\tilde{F}})-\dot{\tilde{\Gamma}}] \odot_{3} \delta_{*} B \mathrm{~d} V \mathrm{~d} t=0
$$

and the weak forms associated with the higher order gradient formulation in $\boldsymbol{C}$ take the form

$$
\begin{aligned}
& \int_{T} \int_{\mathcal{B}_{0}}\left[\boldsymbol{S}: \frac{1}{2} \frac{\partial \dot{\boldsymbol{C}}}{\partial \dot{\boldsymbol{q}}}+\boldsymbol{S}_{G}: \frac{1}{2} \frac{\partial \dot{\boldsymbol{C}}}{\partial \dot{\boldsymbol{q}}}-\dot{\boldsymbol{p}}\right] \cdot \boldsymbol{\delta}_{*} \dot{\boldsymbol{q}} \mathrm{d} V \mathrm{~d} t=0 \quad \int_{T} \int_{\mathcal{B}_{0}}\left[\dot{\tilde{\boldsymbol{C}}}-\dot{\tilde{\boldsymbol{C}}}_{G}\right]: \boldsymbol{\delta}_{*} \boldsymbol{S}_{G} \mathrm{~d} V \mathrm{~d} t=0 \\
& \int_{T} \int_{\mathcal{B}_{0}}\left[\boldsymbol{S}_{G}-\boldsymbol{B} \odot_{3} \frac{\partial \nabla \dot{\tilde{\boldsymbol{C}}}_{G}}{\partial \dot{\tilde{\boldsymbol{C}}}_{G}}\right]: \delta_{*} \dot{\tilde{\boldsymbol{C}}}_{G} \mathrm{~d} V \mathrm{~d} t \quad \int_{T} \int_{\mathcal{B}_{0}}\left[\nabla\left(\dot{\tilde{\boldsymbol{C}}}_{G}\right)-\dot{\tilde{\boldsymbol{\Gamma}}}\right] \odot_{3} \boldsymbol{\delta}_{*} \boldsymbol{B} \mathrm{d} V \mathrm{~d} t=0
\end{aligned}
$$

Obviously, the dependencies for the higher order gradient formulation in $\boldsymbol{C}$ are reduced, since $\Lambda^{\mathrm{C}}$ is dependent only on $\nabla \tilde{\boldsymbol{C}}_{G}$. Hence, many mixed derivations disappear and the tangent becomes substantially simpler.

In the last step, we transform the integrals to a reference element and discretize all quantities over the element in space and time. For the shape functions in space, N, we use Lagrangian 
shape functions (see Reference [3]). For the shape functions in time we use Lagrangian shape functions as well (see Reference [5]), given by

$$
M_{i}(\alpha)=\prod_{\substack{j=1 \\ j \neq i}}^{k+1} \frac{\alpha-\alpha_{j}}{\alpha_{i}-\alpha_{j}}, 1 \leq i \leq k+1 \quad \tilde{M}_{i}(\alpha)=\prod_{\substack{j=1 \\ j \neq i}}^{k} \frac{\alpha-\alpha_{j}}{\alpha_{i}-\alpha_{j}}, 1 \leq i \leq k
$$

The time rate variables and mixed fields $\left(\boldsymbol{q}, \boldsymbol{v}, \boldsymbol{p}, \tilde{\Theta}, \Theta, \eta, \tilde{\boldsymbol{C}}, \tilde{\boldsymbol{C}}_{A}, \tilde{J}, \tilde{\boldsymbol{\Gamma}}, \tilde{\boldsymbol{\Lambda}}, \tilde{\boldsymbol{F}}, \tilde{\boldsymbol{C}}_{G}\right)$ are approximated by

$$
(\bullet)^{e, h}=\sum_{I=1}^{k+1} \sum_{A=1}^{n_{n o}} M_{I}(\alpha) \mathrm{N}^{A}(\boldsymbol{\xi})(\bullet)_{I}^{e A} \quad(\dot{\bullet})^{e, h}=\frac{1}{h_{n}} \sum_{I=1}^{k+1} \sum_{A=1}^{n_{n o}} M_{I}^{\prime}(\alpha) \mathrm{N}^{A}(\boldsymbol{\xi})(\bullet)_{I}^{e A}
$$

and the approximation of Lagrangian multipliers and variation fields $\left(\boldsymbol{\lambda}_{q}, \boldsymbol{S}, \boldsymbol{S}_{A}, p, \boldsymbol{B}, \boldsymbol{H}, \boldsymbol{P}, \boldsymbol{S}_{G}, \boldsymbol{\delta}_{*} \bullet\right)$ takes the form

$$
(\bullet)^{e, h}=\sum_{I=1}^{k} \sum_{A=1}^{n_{n o}} \tilde{M}_{I} \mathrm{~N}^{A}(\bullet)_{I}^{e A}
$$

Here, $n_{n o}$ is the number of nodes of the spatial discretization and $k$ is the polynomial degree in time. With the corresponding Gaussian quadrature rule we approximate each integral. We condense out the resulting formulation a the clement level to a displacement and temperature formulation (see Reference [2]), after gliminating $p$ and $\eta$. Therefore, all mixed fi $q$ and $\Theta$, are discontinuous at the boundaries of Next, the conservation of angular momentum must be corrected, since the higher gradient formulation results in internal moments. If we follow the procedure described in Reference [6],

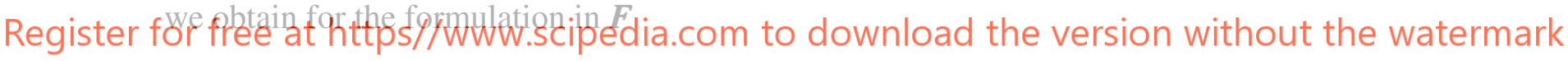

$$
\begin{array}{r}
J_{n+1}-\mathcal{J}_{n}=\int_{t_{n}}^{t_{n}+1} \int_{\mathcal{B}_{0}}\left[\left(\boldsymbol{H}: \frac{\partial \Lambda^{\mathrm{F}}}{\partial \dot{\tilde{\boldsymbol{F}}}}+B \odot_{3} \frac{\partial \nabla \dot{\tilde{F}}}{\partial \dot{\tilde{\boldsymbol{F}}}}\right) \times \tilde{\boldsymbol{F}}\right] \mathrm{d} V \mathrm{~d} t+\int_{t_{n}}^{t_{n}+1} \int_{\partial \mathcal{B}_{0}}\left[\boldsymbol{q} \times \boldsymbol{\lambda}_{q}\right] \mathrm{d} \mathrm{d} \mathrm{d} t \\
+\int_{t_{n}}^{t_{n}+1} \int_{\mathcal{B}_{0}}\left[\boldsymbol{q} \times \boldsymbol{\rho}_{0} \boldsymbol{g}\right] \mathrm{d} V \mathrm{~d} t
\end{array}
$$

and for the formulation in $\boldsymbol{C}$

$$
\begin{aligned}
\mathcal{I}_{n+1}-\mathcal{I}_{n}=\int_{t_{n}}^{t_{n}+1} \int_{\mathcal{B}_{0}}\left[\boldsymbol{B} \odot_{3} \frac{\partial \nabla \dot{\tilde{\boldsymbol{C}}}_{G}}{\partial \dot{\tilde{\boldsymbol{C}}}_{G}} \times \tilde{\boldsymbol{F}}\right] \mathrm{d} V \mathrm{~d} t & +\int_{t_{n}}^{t_{n}+1} \int_{\partial \mathcal{B}_{0}}\left[\boldsymbol{q} \times \boldsymbol{\lambda}_{q}\right] \mathrm{d} A \mathrm{~d} t \\
& +\int_{t_{n}}^{t_{n}+1} \int_{\mathcal{B}_{0}}\left[\boldsymbol{q} \times \rho_{0} \boldsymbol{g}\right] \mathrm{d} V \mathrm{~d} t
\end{aligned}
$$

We use our In-House Matlab code fEMcon based on the implementation and ideas shown in Reference [3]. To solve the linear systems of equations we use the Pardiso solver from Reference [8]. For the assembly procedure we use the fast sparse routine shown in Reference [9]. 


\section{NUMERICAL EXAMPLES}

As numerical example serves a simple cantilever beam which oscillates in a gravitational field. The geometry, configuration and simulation parameters can be found in Figure 1. The corresponding strain energy functions are

$$
\begin{aligned}
& \Psi_{\mathrm{M}}^{\mathrm{iso}}=\frac{\varepsilon_{1}}{2}(\operatorname{tr}[\boldsymbol{C}]-3-2 \ln (J)) \quad \Psi_{\mathrm{M}}^{\mathrm{vol}}=\frac{\varepsilon_{2}}{2}\left(\ln (J)^{2}+(J-1)^{2}\right) \\
& \Psi_{X}^{\mathrm{cap}}=c_{X}^{0}\left(1-\Theta_{\infty} c_{X}^{1}\right)\left(\Theta-\Theta_{\infty}-\Theta \ln \frac{\Theta}{\Theta_{\infty}}\right)-\frac{1}{2} c_{X}^{0} c_{X}^{1}\left(\Theta-\Theta_{\infty}\right)^{2} \\
& \Psi_{\mathrm{F}}^{\mathrm{ela}}=\frac{\varepsilon_{3}}{2}(\operatorname{tr}[\boldsymbol{C M}]-1)^{2} \quad \Psi_{\mathrm{HOG}}^{\mathrm{X}}=l^{2}\left(I_{6}^{\mathrm{X}}\right)^{2}
\end{aligned}
$$
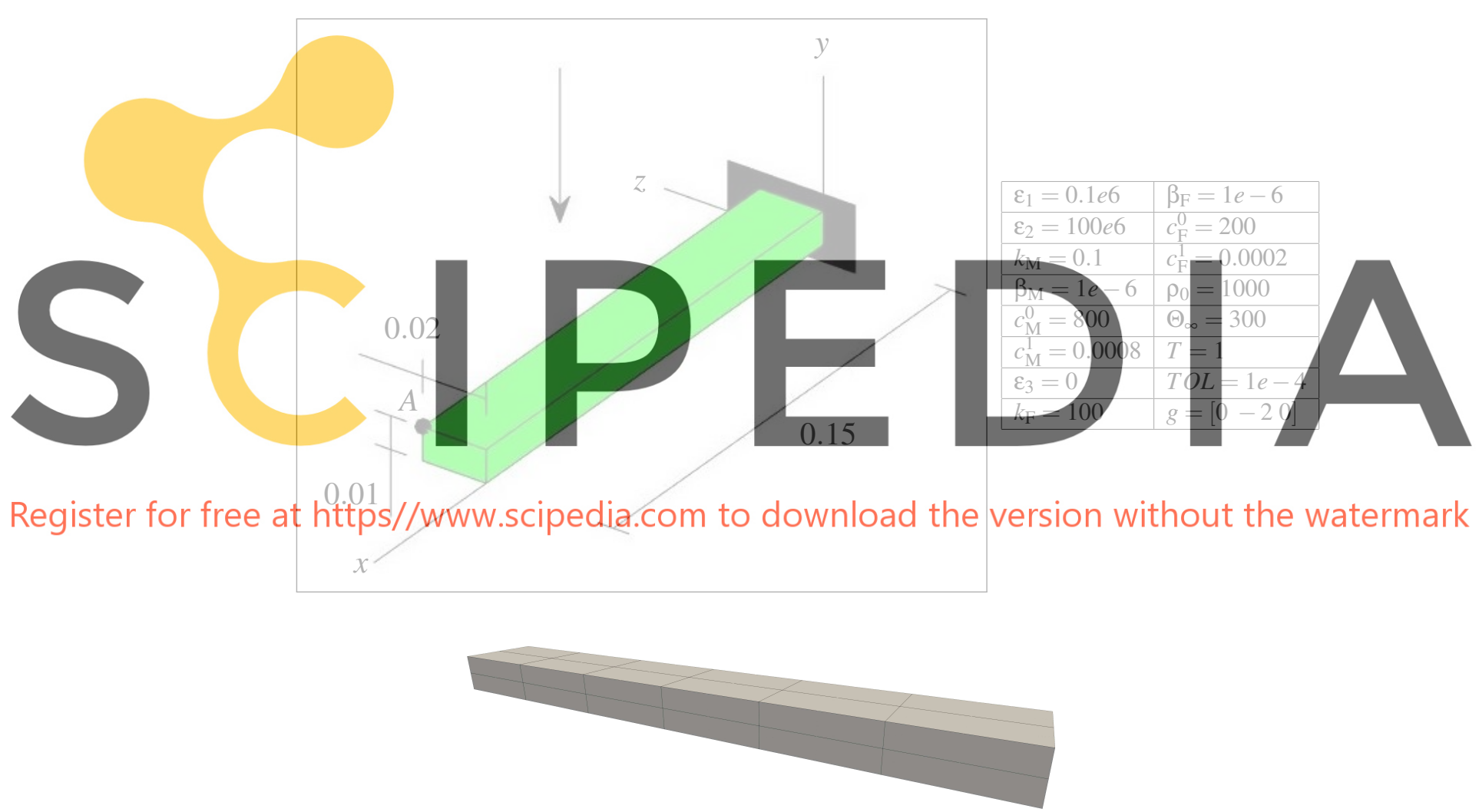

Figure 1: Geometry, configuration and simulation parameters of the cantilever beam for $n_{e l}=24$.

The elastic part of the fiber roving $\Psi_{\mathrm{F}}^{\text {ela }}$ can be found in [7] and for the capacitive part the function $\Psi_{X}^{\text {cap }}$ in Reference [5]. We use an quadratic serendipity mesh (20 nodes) with $n_{e l}=24$. In order to avoid locking we approximate $\tilde{J}$ linear and $\tilde{\boldsymbol{C}}_{A}$ constant. We introduce a length scale parameter $l^{2}$ with $c=\varepsilon_{1} l^{2}$ for the material parameters of $\Psi_{\mathrm{HOG}}^{\mathrm{X}}$. 
First we look in Figure 2 at the influence of the different higher order gradient fomulations. We can see that the $\nabla \boldsymbol{F}$ formulation is stiffening the bending behavior of the beam (red). But the $\nabla \boldsymbol{C}$ formulation also makes this possible, although not to the same level (green). With adjustment of the material parameters, however, we also obtain similar behavior here (blue). As we can see in Figure 3, the angular momentum is perfectly preserved in each variant.

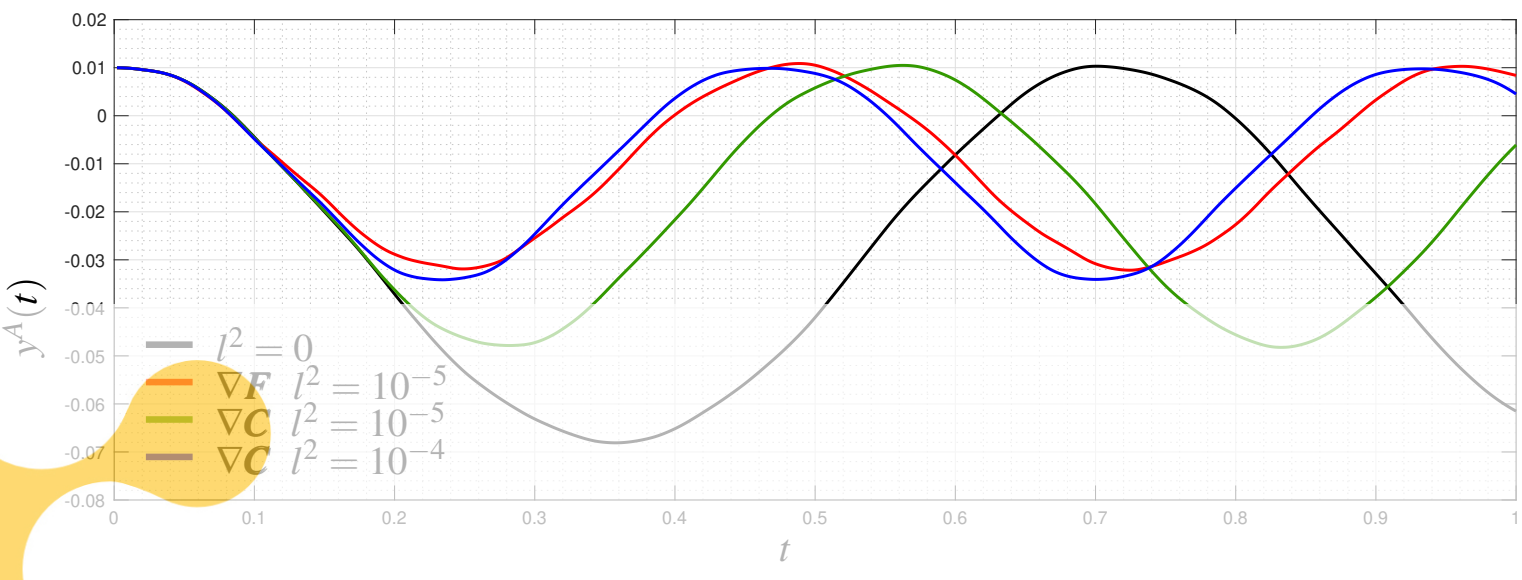

Figure 2: Trajectory of point A for the parameters shown in Figure 1 and the different fomrulations and $\left(\boldsymbol{a}_{0}\right)^{T}=$

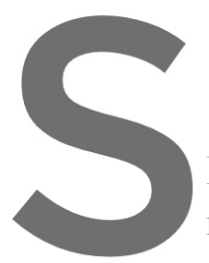

$\left[\begin{array}{lll}1 & 0 & 0\end{array}\right]$

100].

In the next step we set $\Psi_{\mathrm{HOG}}^{\mathrm{X}}$

roving direction is specified here

for three different angles for $\nabla F$
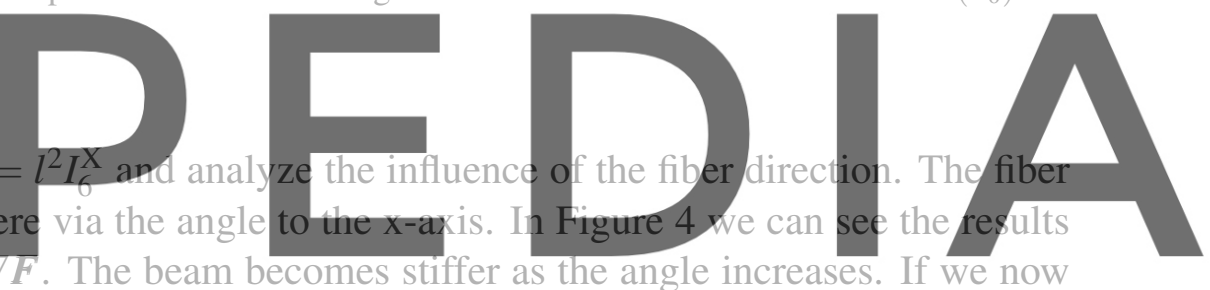

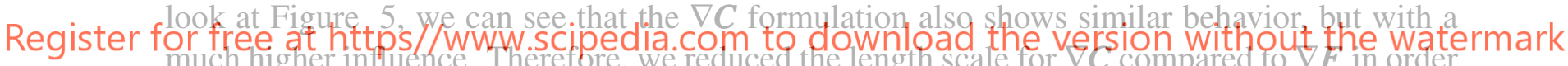
much higher infuence. Therefore, we reduced the length scale for $V C$ compared to $F$ in order

to obtain simular results.

In the last step we take the $\nabla \boldsymbol{C}$ formulation and add a fiber roving stretch stiffness $\Psi_{\mathrm{F}}^{\text {ela }}$. Also we set $\Psi_{\mathrm{HOG}}^{\mathrm{X}}=l^{2}\left(I_{6}^{\mathrm{X}}\right)^{2}$. The resulting trajectory can be found in Figure 6 . As we can see, the additional formulation increases further the stiffness of the beam. We also can see in Figure 7, the energy is perfectly preserved for this case. Finally, we look at the v. Mises equivalent stress $\sigma_{V M}$ and temperature distribution $\Theta$ in Figure 7. On the one hand, we observe the typical stress distribution of a candilever beam with the highest values on the outside, and on the other hand, we see that the fiber roving with its very high thermal conductivity distributes the heat caused by the thermo-mechanical coupling to the whole beam.

\section{CONCLUSIONS}

We could show that a formulation of a higher order gradient based material formulation can be expressd in terms of the right Cauchy-Green tensor, and achieve similar effects as the formulation based on the deformation gradient. This is a remarkable result, because the former 


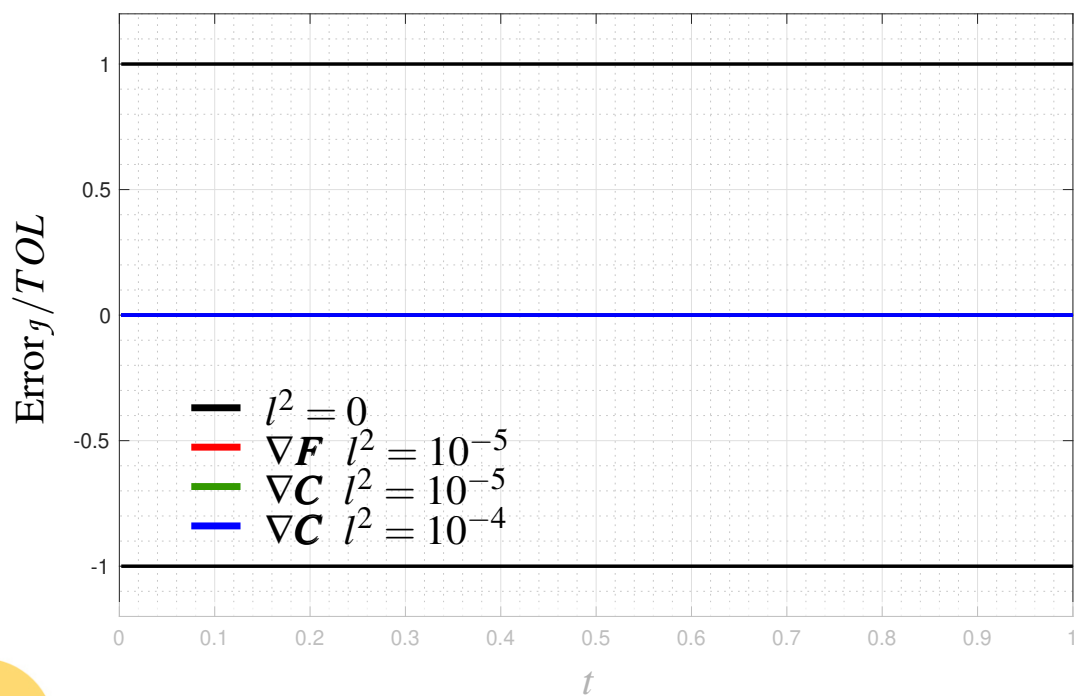

Figure 3: Error of angular momentum $g$ for the parameters shown in Figure 1 and the different fomrulations and $\left(\boldsymbol{a}_{0}\right)^{T}=\left[\begin{array}{lll}1 & 0 & 0\end{array}\right]$.

formulation requires considerably less numerical effort. Also, both formulations work in a thermomechanical contex

tegrators conserve encr

viscous dissipation. In

terms of $\nabla C$ and thus

Acknowledgments
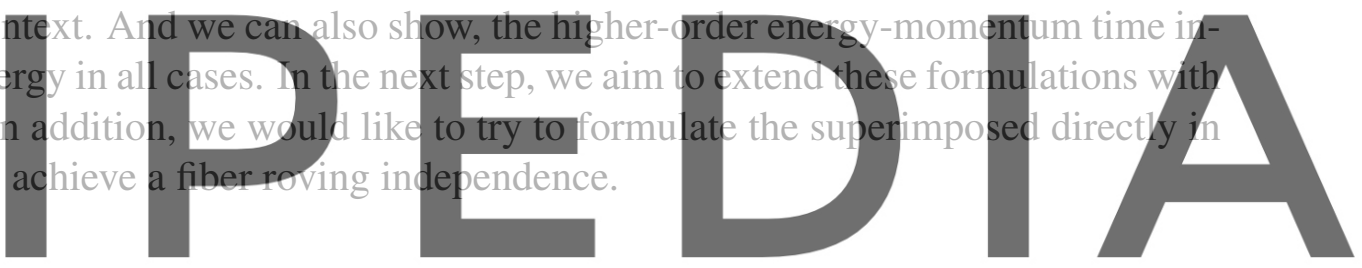

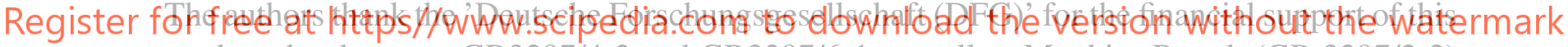
work under the grant GR3297/4-2 and GR3297/6-1 as well as Matthias Bartelt (GR 3297/2-2) for providing the programming basis for the current implementation.

\section{REFERENCES}

[1] Simo, J. C., Taylor, R. L., and Pister, K. S. (1985). Variational and projection methods for the volume constraint in finite deformation elasto-plasticity. Comput. Methods Appl. Mech. Engrg., 51(1-3), 177-208. https://doi.org/10.1016/0045-7825(85)90033-7

[2] Schröder, J., Viebahn, V., Wriggers, and P., Balzani, D. (2016). A novel mixed finite element for finite anisotropic elasticity; the SKA-element Simplified Kinematics for Anisotropy. Comput. Methods Appl. Mech. Engrg., 310:475-494.

[3] Bartelt, M., Dietzsch, J., and Groß, M. (2018). Efficient implementation of energy conservation for higher order finite elements with variational integrators. Math. Comput. Simulat., 150, 83-121. https://doi.org/10.1016/j.matcom.2018.03.002 


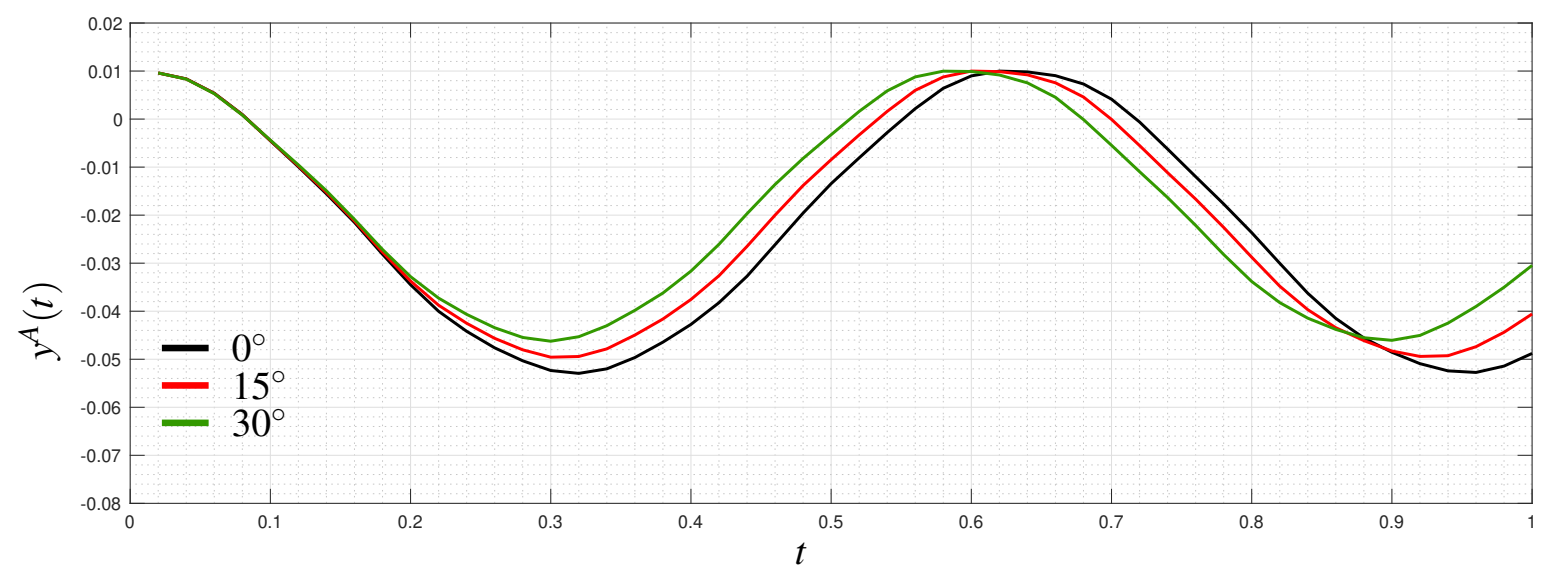

Figure 4: Trajectory of point A for the parameters shown in Figure 1 and the different fiber roving angles for $\nabla \boldsymbol{F}$ and $l^{2}=5 e-6$
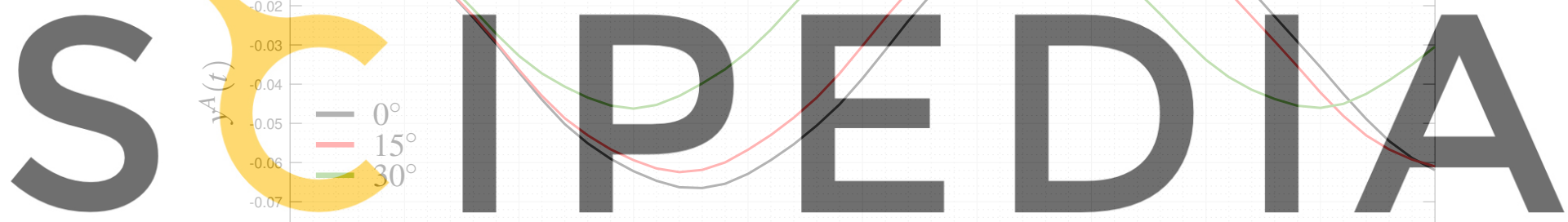

Register for free at https//www.scipedia.com to dównload the version without the watermark

Figure 5: Trajectory of point A for the parameters shown in Figure 1 and the different fiber roving angles for $\nabla C$ and $l^{2}=2.5 e-6$.

[4] J. Dietzsch and M. Groß, Mixed Finite Element Formulations for Polyconvex Anisotropic Material Formulations in WCCM-ECCOMAS2020.

[5] Groß, M., Dietzsch, J., and Bartelt, M. (2018). Variational-based higher-order accurate energy-momentum schemes for thermo-viscoelastic fiber-reinforced continua. Comput. Methods Appl. Mech. Engrg., 336, 353-418. https://doi.org/10.1016/j.cma.2018.03.019

[6] Groß, M., Dietzsch, J., and Röbiger, C. (2020). Non-isothermal energy-momentum time integrations with drilling degrees of freedom of composites with viscoelastic fiber bundles and curvature-twist stiffness. Computer Methods in Applied Mechanics and Engineering, 365, 112973. https://doi.org/10.1016/j.cma.2020.112973

[7] Dal, H., Gültekin, O., Aksu Denli, F., and Holzapfel, G. A. (2017). 


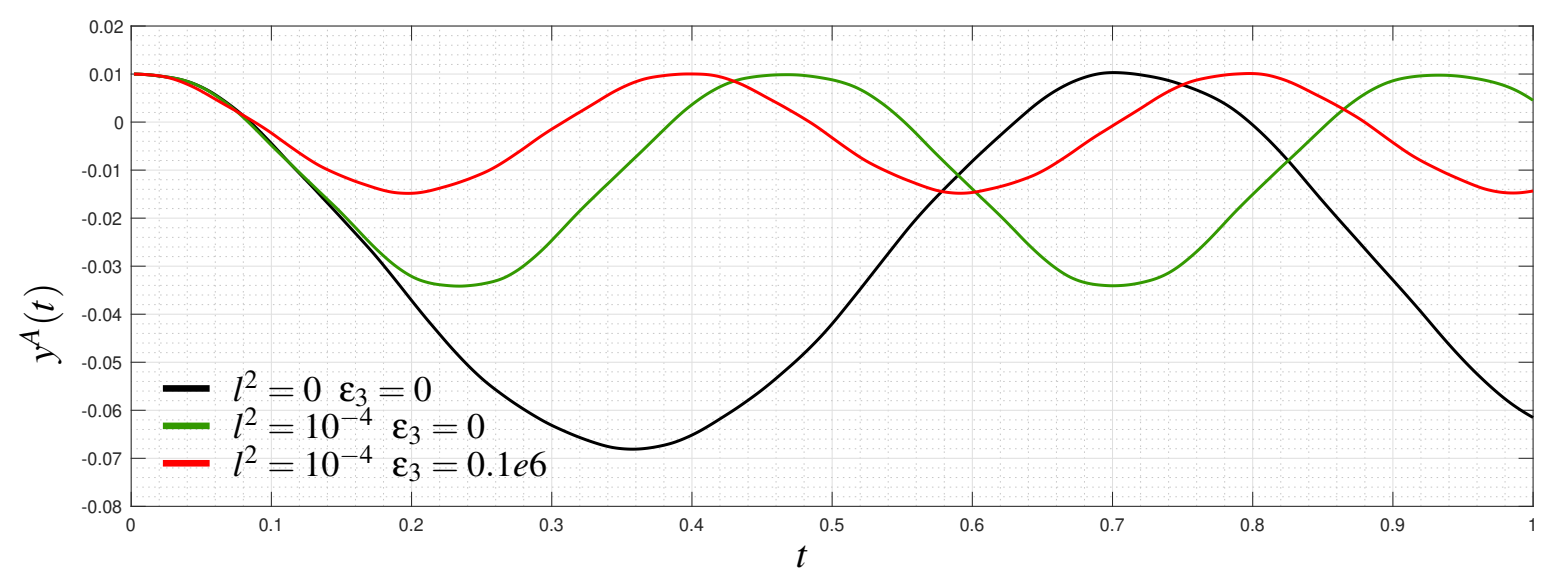

Figure 6: Trajectory of point A for the parameters shown in Figure $1,\left(\boldsymbol{a}_{0}\right)^{T}=\left[\begin{array}{lll}1 & 0 & 0\end{array}\right]$ and $\nabla \boldsymbol{C}$.

Phase-Field Models for the Failure of Anisotropic Continua. PAMM, 17(1). https://doi.org/10.1002/pamm.201710027

[8] Alappat, C., Basermann, A., Bishop, A. R., Fehske, H., Hager, G., Schenk, O., Thies, J., and Wellein, G. (2020). A Recursive Algebraic Coloring Technique for Hardwareefficient Symmetric Sparse Matrix-vector Multiplication. ACM Transactions on Parallel Computing, 7(3). https://doi.org/10.1145/3399732

[9] Engblom, S., and Lukarski, D. (2016). Fast Matlab compatible sparse assembly on multicore computers. Parallel Computing, 56, 1-17. https://doi.org/10.1016/j.parco.2016.04.001

[10] Asmanoglo, T., Menzel., A. (2017) A multi-field finite element approach for the modelling of fibre-reinforced composites with fibre-bending stiffness.

Comput. Methods Appl. Mech. Engrg., 317:1037-1067.

[11] Ferretti, M., Madeo, A., dell'Isola, F., \& Boisse, P. (2014). Modeling the onset of shear boundary layers in fibrous composite reinforcements by second-gradient theory. Zeitschrift Fur Angewandte Mathematik Und Physik, 65(3), 587-612. 


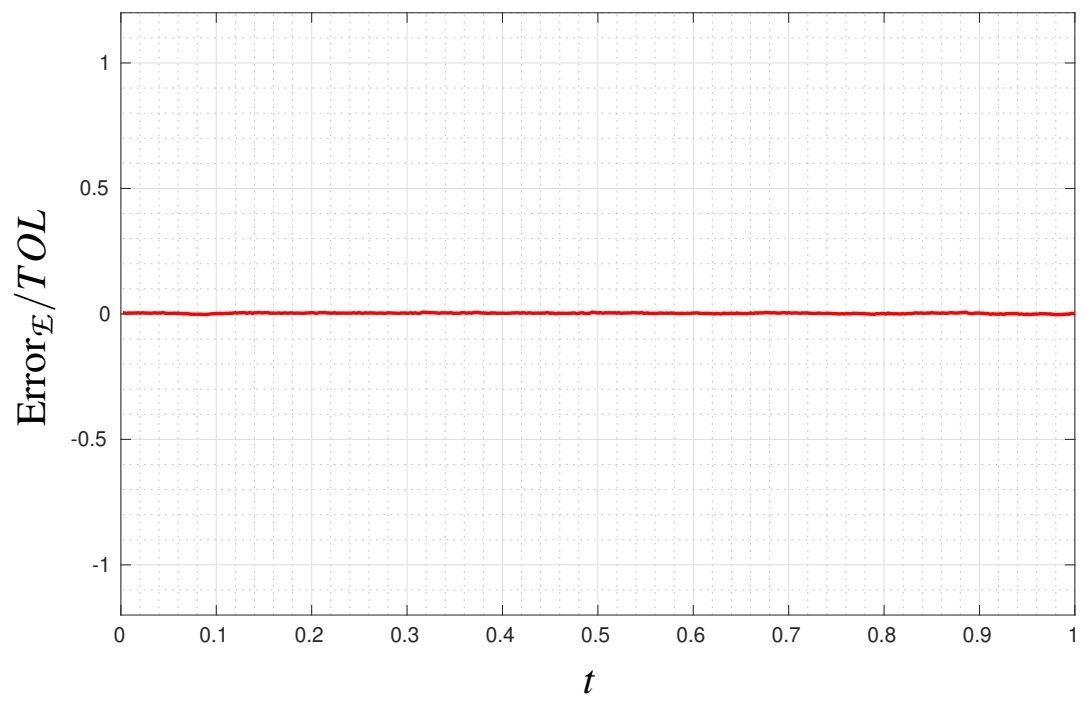

Figure 7: Error of energy $\mathcal{E}$ for the parameters shown in Figure 1 and $\left(\boldsymbol{a}_{0}\right)^{T}=\left[\begin{array}{lll}1 & 0 & 0\end{array}\right]$ for $\nabla \boldsymbol{C}$ and $l^{2}=10^{-4} \varepsilon_{3}=$ $0.1 e 6$.
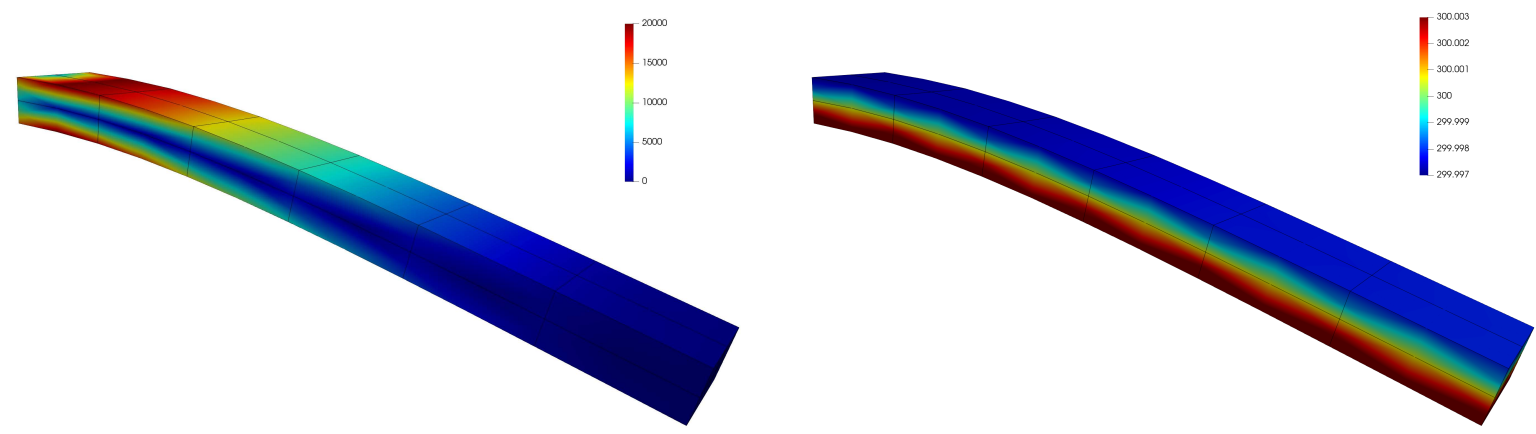

Figure 8: V. Mises equivalent stress $\sigma_{V M}$ and temperature distribution $\Theta$ for the parameters shown in Figure 1 and $\left(\boldsymbol{a}_{0}\right)^{T}=\left[\begin{array}{lll}1 & 0 & 0\end{array}\right]$ for $\nabla \boldsymbol{C}$ and $l^{2}=10^{-4} \varepsilon_{3}=0.1 e 6$ at $t=0.2$ 